\title{
Influence of early environmental factors on peripheral lymphocyte subsets and gut microbiota in infants at risk for celiac disease
}

\author{
T. Pozo-Rubio ${ }^{1}$, G. De Palma ${ }^{2}$, A. Capilla ${ }^{3}$, A. Marcos $^{1}$, Y. Sanz ${ }^{2}$, J. R. Mujico ${ }^{1}$, I. Polanco ${ }^{4}$, \\ M. D. Acuña ${ }^{5}$ and E. Nova ${ }^{1}$ \\ ${ }^{1}$ Immunonutrition Group, ICTAN, CSIC, Madrid, ${ }^{2}$ Microbial Ecophysiology and Nutrition Group, IATA, CSIC, Valencia, \\ Spain, ${ }^{3}$ Biomedicine Institute of Valencia, CSIC, Valencia, ${ }^{4}$ Gastroenterology Service, Hospital La Paz, Madrid, Spain and \\ ${ }^{5}$ Gastroenterology Unit, Hospital Niño Jesús, Madrid, Spain
}

\begin{abstract}
Genetic risk linked to the HLA (Human Leucocyte Antigen) system is not enough to explain the incidence of celiac disease (CD) and other genetic and environmental factors have been suggested to play a role ${ }^{(1)}$. The immune system and microbiota are not fully developed at birth and single antigen encounter in the intestine is a key process to achieve full development ${ }^{(2)}$. Environmental factors through this or other route are believed to impact on the immune system and microbiota as well. We sought to assess the effect of several early environmental factors on lymphocyte subsets and microbiota composition in infants at familial risk for CD. For this purpose, 55 infants with a first degree relative suffering CD were recruited before birth. Information on early environmental factors was prospectively collected including type of delivery, mother's antibiotic intake during pregnancy, mother's antibiotic administration during labour, milkfeeding practices, infections and antibiotic intake in the first 4 months of life and rotavirus vaccine administration. Lymphocyte subsets in peripheral blood and gut microbiota composition in faeces samples were studied at the age of 4 months by flow cytometry analysis and qPCR respectively. Linear regression analysis showed that, the absolute counts of total lymphocytes, CD3,$+ \mathrm{CD} 4+, \mathrm{CD} 4+\mathrm{CD} 38+$, CD4 + CD28+, were positively associated with formula-fed infants and infant's infections in the first 4 months of age. The percentage of $\mathrm{CD} 4+\mathrm{CD} 25+$ was positively associated with vaginal delivery and antibiotic use by mothers during pregnancy, and negatively with rotavirus vaccine, and their absolute counts were associated positively with infant's infections. The absolute counts of $\mathrm{CD} 3+\mathrm{CD} 4+\mathrm{CD} 45 \mathrm{RO}+$ were positively associated with formula-feeding and infant's infections, and negatively with infant's antibiotic intake. CD4 + HLA-DR + cell counts were positively associated with infant's infections. The percentages and absolute counts of NK cells were positively associated with infant's infections and antibiotic administration in mothers during labor. Regarding microbiota, infant's antibiotic intake is associated with higher counts of Bacteroides spp. and lower counts of $B$. longum. Cesarean delivery is associated with higher counts of $B$. angulatum and lower counts of B. catenulatum. B. angulatum counts were also positively associated with infant's antibiotic administration in the first 4 months of life and negatively with formula feeding and antibiotic administration during pregnancy. In conclusion, the balance between the effects of the pre- and post-natal factors on lymphocyte subsets and microbiota composition might modify the risk for future development of immune-related diseases such as celiac disease.
\end{abstract}

This work was supported by grants AGL2007-66126-C03-01/ALI and AGL2007-66126-C03-03/ALI from the Spanish Ministry of Science and Innovation. T. Pozo-Rubio is recipient of a JAE-CSIC grant co-funded by European Social Fund.

1. Dubois PC \& van Heel DA (2008) Clin Exp Immunol 153, 162-173.

2. Williams AM, Bland PW, Phillips AC et al. (2004) J Immunol 173, 7190-7199. 